\title{
Systems biology approaches based discovery of a small molecule inhibitor targeting both c-Met/PARP-1 and inducing cell death in breast cancer
}

\author{
Tian $\mathrm{Yu}^{1^{*}}$, Lijia Cheng ${ }^{1 *}$, Xueling Yan ${ }^{1}$, Hang Xiong ${ }^{1}$, Jie Chen ${ }^{3}$, Gang He${ }^{1}$, Hui Zhou ${ }^{1}$, Hongbo Dong1, \\ Guangya Xu1 ${ }^{1}$, Yong Tang ${ }^{1,2}{ }^{凶}$, Zheng Shi ${ }^{\bowtie}$ \\ 1. School of Medicine \& Sichuan Industrial Institute of Antibiotics \& Department of Respiratory and Critical Care Medicine, Affiliated Hospital/ Clinical \\ College of Chengdu University, Chengdu University, Chengdu 610015, China \\ 2. School of Acupuncture and Tuina, Chengdu University of Traditional Chinese Medicine, Chengdu 610075, China \\ 3. Central Laboratory of Clinical Medicine, Sichuan Academy of Medical Science and Sichuan Provincial People's Hospital, Chengdu, 610000, China \\ *Co-first authors, who contributed equally. \\ $\triangle$ Corresponding authors: Dr. Yong Tang \& Dr. Zheng Shi, School of medicine, Chengdu University \& School of Acupuncture and Tuina, Chengdu University \\ of Traditional Chinese Medicine, Chengdu, Sichuan 610016, P.R. China, E-mail: tangyong@cdutcm.edu.cn \& drshiz1002@hotmail.com
}

(c) The author(s). This is an open access article distributed under the terms of the Creative Commons Attribution License (https://creativecommons.org/licenses/by/4.0/). See http://ivyspring.com/terms for full terms and conditions.

Received: 2019.09.29; Accepted: 2020.01.28; Published: 2020.02.19

\begin{abstract}
Breast cancer is the second most common types of cancer worldwide. Molecular strategies have developed rapidly; however, novel treatments strategies with high efficacy and lower toxicity are still urgently demanded. Notably, biological networks estimated from microarray data and functional activity network analysis could be utilized to identify and validate potential targets. In this study, two microarray data (GSE13477, GSE31192) were firstly selected, and analyzed by multi-functional activity network analysis to generate the core protein-protein-interaction (PPI) network. Several potential targets were subsequently identified and c-Met and poly (ADP-ribose) polymerase-1 (PARP-1) were manually chosen as the key targets in breast cancer. Furthermore, virtual screening and molecular dynamics (MD) simulations were utilized to recognize novel c-Met/PARP-1 inhibitors in Specs products database. Three small molecules, namely, ZINC19909930, ZINC20032678 and ZINC13562414 were selected. Additionally, these compounds were synthesized, and two breast cancer cell lines, MDA-MB-231 and MCF-7 cells were used to validate our bioinformatic findings in vitro. MTT assay and Hoechst staining showed that ZINC20032678 significantly induced breast cancer cell death, which was mediated through apoptosis by flow cytometry. Furthermore, ZINC20032678 was shown to target the active sites of the both targets and recruitment of downstream apoptotic signaling pathways, eventually inducing breast cancer cell apoptosis. Collectively, our findings not only offer systems biology approaches based drug target identification, but also provide the new clues for developing novel inhibitors for future breast cancer research.
\end{abstract}

Key words: breast cancer, c-Met, PARP-1, systems biology, apoptosis, drug discovery

\section{Introduction}

Breast cancer is one of the most leading causes of cancer death among women. It is reported that the overall pathogenic incidence rate in women remains generally stable, whereas the breast cancer incidence has slight increased from 2004 to 2013 [1].Currently, there are four main molecular subtypes of breast cancer, referring as luminal, HER2, normal-like and basal. Of note, targeted therapies have been raised 
great attention in science community. It can target specific targets expressed in/on the surface of cancer cells which are involved in carcinogenesis and/or tumor growth [2]. For example, tamoxifen for treatment of ER positive tumors and trastuzumab in the treatment of HER2 positive tumors in breast cancer have been extensively studied [3]. However, it is worth knowing that most basal-like breast cancers do not express ER, PR and HER2, the two subgroups are not mutually exclusive. And, $80 \%$ of basal-like breast cancers are triple-negative and $80 \%$ of triplenegative breast cancers exert a basal-type phenotype [4]. Therefore, fundamental breakthroughs, particularity new therapeutic targets and new drugs are most urgent needed in breast cancer research.

It is known to all that the entire DNA microarrays has been used in cancer drug development and facilitate clinical applications for years [5]. Since measurement of the sequence and expression of potential targets is greatly facilitated by microarray technology, it is of great importance to generate new clues to gene function which can help to identify appropriate targets for therapeutic intervention. Hitherto, a great deal of drug targets discovery and validation have been well described by microarray analysis [6, 7]. Additionally, DAVID online database, an integrated biological knowledge base and analytic tool, can extract biological meaning from large gene/protein lists [8]. Moreover, STRING database includes known and predicted PPIs which stem from various databases [9]. Combining the microarray data and functional activity network analysis of differentially expressed genes by using DAVID (including KEGG pathway and GO analysis) and STRING were accelerate the drug target development [10]. To our knowledge, a number of hub proteins/targets were successfully identified by using abovementioned approaches [11, 12].

In this study, two microarray data (GSE13477, GSE31192) were firstly analyzed to obtain consensus results of differently expressed genes. Then, the upregulated genes were selected to identify the candidate genes by using GO, KEGG pathway and STRING analysis. According to the network results and consulting numerous references, c-Met and PARP-1 were manually selected as the key targets in breast cancer. Subsequently, molecular docking and MD simulations were performed to recognize valuable agents to target both c-Met/PARP-1. Then, three small molecules, namely ZINC13562414, ZINC20032678 and ZINC19909930 were identified as potential inhibitors against both c-Met and PARP-1. We further synthesized three compounds, and cultured MDA-MB-231 and MCF-7 cells and treated with these compounds. Eventually, the compound
ZINC20032678 effectively induced breast cancer cell apoptosis through inhibition of both c-Met and PARP-1 kinases activities. In general, our findings would lead to comprehensive mechanistic insights into identification of more ideal targets as well as more novel potential drugs in future cancer drug discovery.

\section{Material and methods}

\section{Hub protein identification}

In order to investigate if genes were co-expressed or not, two microarray data (GSE13477; GSE31192) under the determiners "Breast cancer" AND "Homo sapiens" from the GEO Data Sets were selected, and analyzed by using GEO2R tool (https://www.ncbi.nlm.nih.gov/geo/geo2r/). Two microarray data by using different text conditions were selected. The aim of two chips is to identify differential genes between normal tissues and cancer (cells), while excluding the error of individual chip experiments through cell chips and tissue chips. GO analysis could provide comprehensive information on gene function of individual products through ontology. An Expression Analysis Systematic Explorer (EASE) score $<0.1$ was adopted to refine the GO terms set in major clusters through Database for Annotation, Visualization and Integrated Discovery Bioinformatics Resources version 6.8 (DAVID:https://david.ncifcrf.gov/). A protein interaction network of each gene and all protein interaction networks of up-regulated genes and down-regulated genes were constructed through STRING. Subsequently, the selected genes were carried up by using DAVID to get the KEGG pathway and Gene Ontology results, and using STRING to get the core PPI network, respectively. We further imported the results into cytoscape to strip out the core PPI network. Herein, the identification of the hub protein schematic model was shown in Figure 1.

\section{Molecular docking}

The initial 3D X-ray crystal structures of c-Met (3ZCL) and PARP-1 (3L3M) were downloaded from the RCSB Protein Data Bank (PDB) (http:/ / www.pdb. org/pdb/home/home.do) [13]. 32791 candidate compounds that were commercially available for screening were collected from specs subsets (http:// zinc.docking.org/catalogs/specsnp) [14]. The c-Met/ PARP-1 and their ligands were prepared by USCF chimera (Version1.8) by elimination of solvent molecules as well as insertion of hydrogen atoms and standard charges [15]. UCSF DOCK (version 6.5) was used to identify compound libraries against a target receptor to predict potential drugs $[16,17]$. To ensure the accuracy of docking, the specified parameters 
docking were confirmed by redocking the co-crystallized ligand back into the binding site of the receptor. Root mean square deviation (RMSD) serves as a reference value for judging docking performance $[18,19]$. The top 100 ranked small molecular natural products were rescored by amber score function [20, 21]. Generally speaking, the lower score of ligands indicates the higher binding affinity to receptor.

\section{MD simulations}

GROMACS package (Version 4.5) [22] was adopted to perform the MD simulations. Amber ff99SB force field and TIP3P water mode were explored to convert the protein structure into the topology [23]. Additionally, the ligands were charged by AM1-BCC via antechamber program. Then, parmchk program was utilized to check and revise the missing force field parameters as well as converting the parameters and topologies for ligands with ACPYPE tools [24]. Simulations were carried out under periodic boundary conditions (PBC) with a dodecahedron periodic box setting the minimal distance of $1.0 \mathrm{~nm}$ between the protein and edge of the box, which was solvated by adding simple point charge (SPC) water molecules [25]. Moreover, $\mathrm{Na}^{+}$ and $\mathrm{Cl}^{-}$were added to mimic a physiological $\mathrm{NaCl}$ concentration of $0.15 \mathrm{M}$ to neutralize the solvated system. Subsequently, we employed the steepest descent minimization algorithm for all atoms to minimize the energy of system [26]. Afterwards, 100 ps NVT (constant number of particles, volume, and temperature) and 100 ps NPT (constant number of particles, pressure, and temperature) equilibrations were performed for each system with position restraints for receptor and potent small molecules. In the end, $25 \mathrm{~ns}$ MD simulations with a time step of $2 \mathrm{fs}$ at constant pressure $(1 \mathrm{~atm})$ and temperature $(300 \mathrm{~K})$ were performed. During the MD simulation process, all bond lengths were constrained by LINCS algorithm and Particle Mesh Ewald (PME) method was adopted to calculate the long-range electrostatic interactions [27-29]. The trajectories of c-Met/PARP-1 bounded with different small molecules were computed by backbone root mean square deviation (RMSD, g_rms) of GROMACS utilities.

\section{Chemical synthesis of candidate compounds}

Synthesis of ZINC20032678 requires two key intermediates a4 and dodecane-1, 12-diamine, which were synthesized using the route described in Figure 2A (ZINC20032678). Dodecane-1, 12-diamine was commercial availability. To prepare the intermediates a4, compound a1 was treated with $\mathrm{CS}_{2}$ and heated in the water to furnish the corresponding carbamodithioic acid a2. Compound a2 was oxidized by $\mathrm{HgCl}_{2}$ to afford ethyl, 2-isothiocyanatoacetate a3. Finally intermediate a4 could be obtained from a3 through cyclization in DMF at $120 \mathrm{C}$.

For ZINC19909930, our synthetic strategy started from commercially available 3-bromopropan-1-ol b1.Compound b1 was treated with phenol and then 1 , 4-dibromobut-2-yne to get key Intermediate b3. Compound b3 was reacted with piperazine to get ZINC19909930 in good yield (Figure 2B).

As for compound ZINC13562414, our synthetic strategy started from compound c1. Compound c1 was reacted with 1-(4-nitrophenyl) ethan-1-one to get compound $\mathbf{c 2}$. The amino hydrogens of compound $\mathbf{c 2}$ were replaced by benzyl groups to get ZINC13562414 (Figure 2C).
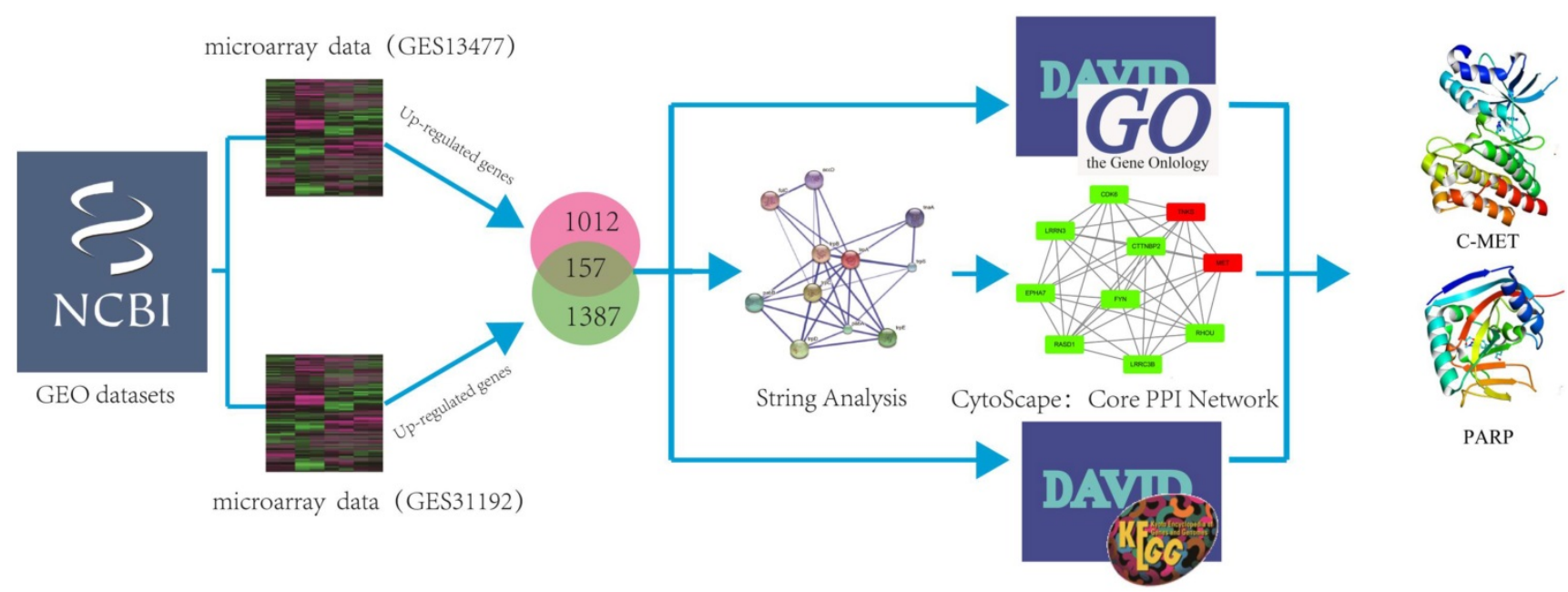

Figure 1. The schematic model of data mining and virtual screening process. We systematically combined two microarray datasets and analyzed these data by using GO and pathway enrichment analysis to predict hub proteins in breast cancer. 
A

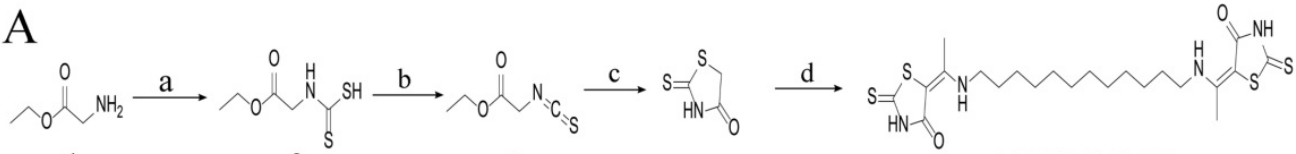

a1

a2

a3

a4

ZINC20032678

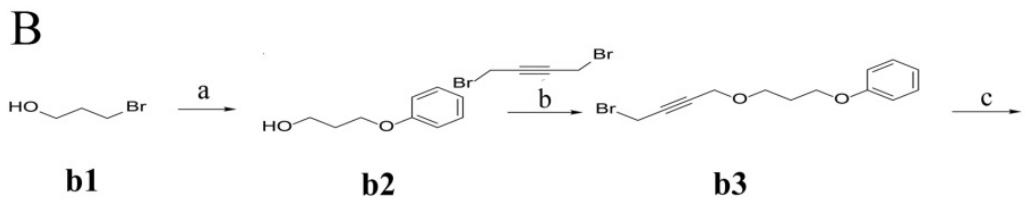

C<smiles>CN1C(=O)NC(=O)C2NC(NN)=NC21</smiles>

c1

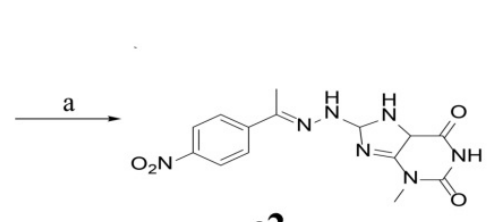

c2

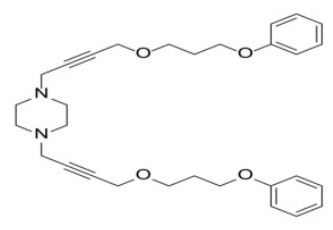

ZINC19909930

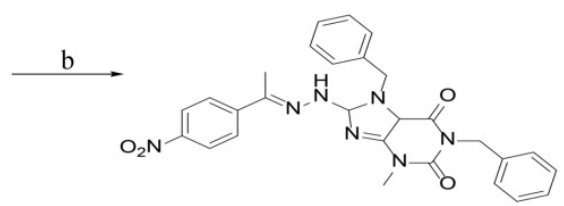

ZINC13562414

Figure 2. Synthetic route of three compounds. (A) Synthetic Approach to $\mathrm{ZINC20032678}$. Reagent and conditions: (a) $\mathrm{CS}_{2}$, water, ambient temperature, $48 \mathrm{~h}$; (b) $\mathrm{HgCl}_{2}$, methylbenzene, $5 \mathrm{~h}$; (c) DMF, $120^{\circ} \mathrm{C}$, 6h; (d) $\mathrm{CH}_{3} \mathrm{CHO}, \mathrm{NH}_{2}\left(\mathrm{CH}_{2}\right){ }_{12} \mathrm{NH}_{2}$, 6h. (B) Synthetic Approach to ZINC19909930. Reagent and conditions: (a) 18-crown-6-ether, $\mathrm{K}_{2} \mathrm{CO}{ }_{3}$, acetone, 12h; (b) $\mathrm{NaOH}, 1.4$-dibromobut-2-yne, N-Benzyl-N-triethylammonium chloride, benzene, $70^{\circ} \mathrm{C}, 15 \mathrm{~h}$; (c) piperazine, $\mathrm{NaOH}$, acetone. (C) Synthetic Approach to ZINC13562414. Reagent and conditions: (a) 1-(4-nitrophenyl)etana-1-one, $\mathrm{KOH} / \mathrm{K}_{2} \mathrm{CO}_{3}$, Water, $0.5 \mathrm{~h}$; (b) (bromomethyl)benzene, $\mathrm{NaOH}$, DMF, 4h.

\section{Cell culture}

The MDA-MB-231 and MCF-7 cells were purchased from American Type Culture Collection (ATCC, Manassas, VA, USA). The cells were cultured in Dulbecco's Modified Eagle Medium (DMEM) with $10 \%$ fetal bovine serum (FBS) and $1 \%$ penicillin/ streptomycin, and maintained in a $5 \% \mathrm{CO}_{2} / 37^{\circ} \mathrm{C}$ incubator, respectively. Cells were using with $0.25 \%$ trypsin, DMEM, FBS and antibiotics were purchased from GIBCO (Carlsbad, CA, USA).

\section{(4,5-dimethyl-2-thiazolyl)-2,5-diphenyltetrazol ium bromide (MTT) assay}

$5 \times 10^{3}$ MDA-MB-231 and MCF-7 cells were seeded in 96-well plate, respectively. Eighteen hours post-seeding, cells were treated with different concentrations of ZINC20032678 as followings: 0.1 $\mu \mathrm{mol} / \mathrm{L}, 1 \mu \mathrm{mol} / \mathrm{L}$ and $10 \mu \mathrm{mol} / \mathrm{L}$ ZINC20032678. Cisplatin was used as positive control, and untreated cells were set as blank control. Each group was set three wells. Forty-eight hours post-drug treatments, the viable cells were stained by adding $20 \mu \mathrm{l}$ of 5 $\mathrm{mg} / \mathrm{ml}$ MTT solution per $100 \mu \mathrm{l}$ of growth medium. After incubating for $4 \mathrm{~h}$ at $37^{\circ} \mathrm{C}$, the media were removed and $150 \mu \mathrm{D}$ DSSO was added to dissolve the formazan. The absorbance of each well was measured by microplate reader and viable cells are presented as a percent of the control.

\section{Hoechst staining}

$1 \times 10^{5}$ MDA-MB-231 and MCF-7 cells were seeded in 6-well plate, respectively. The cells were treated with different concentrations of small molecule ZINC20032678 as followings: $0.1 \mu \mathrm{mol} / \mathrm{L}, 1$ $\mu \mathrm{mol} / \mathrm{L}$ and $10 \mu \mathrm{mol} / \mathrm{L}$ ZINC20032678 after $18 \mathrm{~h}$. Cisplatin was used as positive control and untreated cells were set as blank control. Each group was set three wells. Forty-eight hours later, cells were stained with Hoechst kit from Beyotime (Haimen, Jiangsu, China). Cell counting was carried out using the software ImageJ from National Institutes of Health, which is available at http://rsbweb.nih.gov. The corresponding cell death rates were calculated according to Hoechst staining.

\section{Annexin V-FITC/PI stained fluorescence- activated cell sorter (FACS)}

To explore whether the cell death was caused by apoptosis, MDA-MB-231 cells was chosen to perform Annexin V-FITC/PI stained FACS. After incubation of cells with $0.1,1$ and $10 \mu \mathrm{mol} / \mathrm{L}$ ZINC20032678 for $48 \mathrm{~h}$ in 6-well plate, the cells were harvested through trypsinization, and washed twice with PBS. The cells were centrifuged at $3000 \mathrm{r} / \mathrm{min}$ for $5 \mathrm{~min}$, then the supernatant was discarded and the pellet was resuspended in $1 \times$ binding buffer at a density of $1.0 \times 10^{5} / \mathrm{ml}$. The sample solution of $100 \mu \mathrm{l}$ was transferred to a $5 \mathrm{ml}$ culture tube, and incubated with $5 \mu \mathrm{l}$ FITC-conjugated annexin V (Abcam, USA) and 5 $\mu \mathrm{l}$ PI (Abcam, USA) for $15 \mathrm{~min}$ at room temperature in the dark. $400 \mu \mathrm{l} 1 \times$ binding buffer was added to each sample tube, and the samples were analyzed by FACS (Mindray, China).

\section{Western blot}

Proteins were extracted from MDA-MB-231 cells treated with 0, 0.1, 1 and $10 \mu \mathrm{mol} / \mathrm{L}$ ZINC20032678 
and $1 \mu \mathrm{mol} / \mathrm{L}$ crizotinib (c-Met inhibitor) or NMSP118 (PARP-1 inhibitor) at $72 \mathrm{~h}$, respectively, and the untreated cells were set as the blank control. Then we further separated by using a $10 \%$ polyacrylamide gel. After transferring the protein on a nitrocellulose membrane, the membrane was blocked with a 5\% defatted milk solution and probed with recombinant monoclonal antibody against phosphorylated c-Met (1:2000, Abcam, USA), full-length monoclonal PARP-1 (1:1000, Abcam, USA), cleaved PARP-1 (1:1000, Abcam, USA), cleaved caspase-3 (1:500, Abcam, USA), Bcl2 (1:2000, Abcam, USA), Bax (1:2000, Abcam, USA) and $\beta$-actin (1:5000, Abcam, USA), and then probed with a secondary antibody using ALP conjugated anti-human IgG (1:5000, Santa cruz, USA), at last, blots were developed using a ECL plus kit (GE, USA).

\section{Statistically analyses}

Data are expressed as means \pm standard deviation $(\bar{X} \pm s)$ and were analyzed by ANOVA analysis (SPSS 22.0, USA). A $P<0.05$ was considered statistically significant.

\section{Results}

\section{Identification of hub proteins in breast cancer}

According to GEO datasets, 1169 up-regulated genes and 1544 up-regulated genes were subsequently obtained under the accession number GSE 13477 and GSE 31192, respectively. To avoid false positive results, the consensus results including 157 up-regulated genes were finally selected. These up-regulated genes were analyzed by using DAVID 6.7 to get results of GO analysis and KEGG-pathway analysis, respectively. Genes with $P$ value $(P<0.0054)$ were chosen from of GO analysis, and genes with minimum $P$ value were also selected from KEGG-pathway analysis. At the same time, we analyzed the 157 up-regulated genes by using STRING database to obtain PPI network, then peeling sub-network to obtain core PPI network by using cytoscape software, and genes which had the maximal degree value were eventually chosen. And, GO enrichment analysis and comprehensively integration of results from GO analysis, pathway enrichment and cytoscape software analysis, the core PPI sub-network was established (Figure 3). Hub proteins, such as c-Met, PARP-1, EGFR, IGF1, DASD1 were finally recognized (Table 1). After consulting numbers of references, c-Met and PARP-1 were considered as the key targets in breast cancer.

\section{Virtual screening for potential c-Met/PARP-1 inhibitors}

32791 compounds from specs chemistry database were launched to recognize both c-Met and
PARP-1 inhibitors by virtual screening. After the docking procedure, the detailed information of Top 10 ideal results was shown in Table 2. Subsequently, we finally chose 5 small molecules, namely ZINC1356 2414, ZINC20032678, ZINC19909930, ZINC19909927, and ZINC06444965 for MD simulations to evaluate the dynamic interactions between c-Met/PARP-1 and their potential ligands within the given period of time.

Table 1. The detailed information of hub proteins in breast cancer

\begin{tabular}{ll}
\hline Gene name & Protein name \\
\hline TNKS & Poly [ADP-ribose] polymerase tankyrase-1 \\
CCND2 & G1/S-specific cyclin-D2 \\
ITGA8 & Integrin alpha-8 \\
MET & Hepatocyte growth factor receptor \\
LAMA1 & LAMA1 protein \\
IGF1 & IGF1 protein \\
JUN & Transcription factor AP-1 \\
ITGA1 & Integrin alpha-1 \\
RASD1 & Dexamethasone-induced Ras-related protein 1 \\
RHOU & Rho-related GTP-binding protein RhoU \\
\hline
\end{tabular}

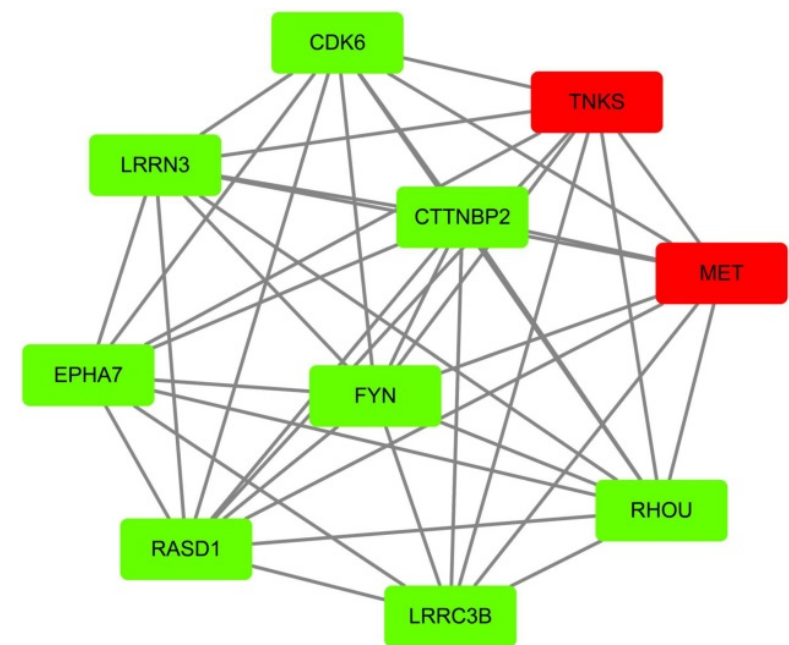

Figure 3. Identification of core PPI network in breast cancer. Co-expressed network of up-regulated differently expressed genes presented by node and edge by the analyzer of cytoscape. The red nodes indicate more protein that could interact with this node, while the green node indicates less protein integrators.

\section{MD simulations}

RMSD value is an important indicator of stability of drug-target interaction. Herein, the backbone RMSD values were calculated to assess the stability of the protein-ligand binding models during the MD simulations. We took $25 \mathrm{~ns} \mathrm{MD}$ simulations to evaluate the dynamic interactions between two targets and their ligands. As was shown in Figure 4A, the 5 selected small molecules had backbone RMSD values ranging from 0.075 to $0.20 \mathrm{~nm}$. Given their starting structures, c-Met-ZINC19909930 and c-MetZINC13562414 showed similarly sharp rise during the 
first 2.5ns. ZINC20032678 reached equilibrium after the initial period of fluctuation with the values of backbone RMSDs around $20 \mathrm{~nm}$. c-Met-ZINC 06444965 system had the trend of rise within $5 \mathrm{ns,}$ while the c-Met-ZINC19909927 system exhibited maximum deviation. Compared to other systems, ZINC13562414, ZINC20032678, ZINC19909930 systems were relatively balanced, indicating these three small molecules had stronger affinities, towards c-Met. Similarly, Figure 4B indicated that PARP-1ZINC19909930, ZINC13562414 and ZINC20032678 systems exerted relatively balance than the other two systems. Therefore, these three molecules were selected for further in vitro study.

\section{Effects of compounds on breast cancer cell viability}

To determine whether the treatment of three compounds affected the cell survival abilities, we examined the cell viability by MTT assay after drug treatment for $48 \mathrm{~h}$ post-drug treatments. Results demonstrated that the cell survival abilities of both MDA-MB-231 and MCF-7 cells were significantly decreased only in ZINC20032678 treated group $(P<0.05)$, not in the other two compound-treatment groups. The effects of ZINC20032678 were similar to that of the positive control group (Figure 5A).
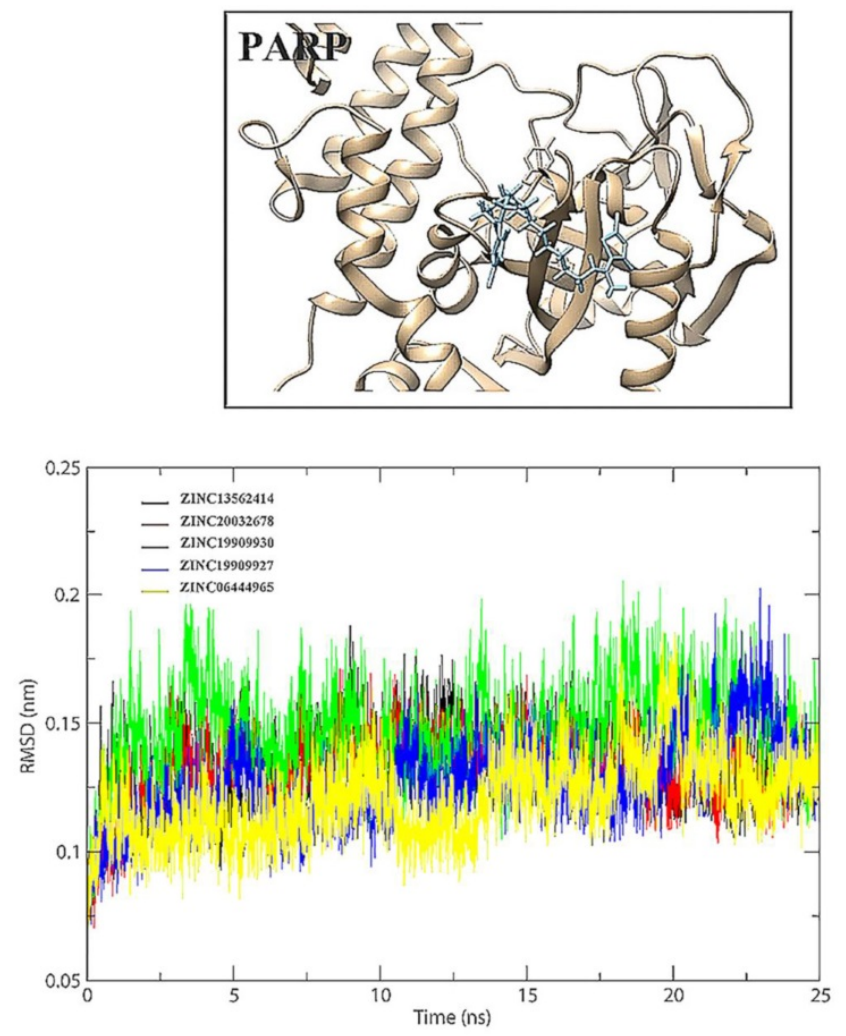

A
Interestingly, such effect was also in a dosedependent manner. These results indicated that the ZINC20032678 could effectively inhibit the breast cancer cell survival.

To further investigate the effects of ZINC 20032678 on the cell survival rate, we investigated the percentages of cell death of the two cell lines, upon ZINC20032678 treatment by Hoechst staining. Compared to the untreated group, the percentage of dead cells was significantly increased in group treated with $10 \mu \mathrm{mol} / \mathrm{L}$ ZINC20032678 and cisplatin (Figure $5 \mathrm{~B} \& \mathrm{C})$. And the effect of inhibiting cell activity was better in MDA-MB-231 cells. Therefore, we chose MDA-MB-231 cells to perform the further study.

Effects of ZINC20032678 on apoptosis of breast cancer cells

To identify the cause of the breast cell death, the Annexin V/PI staining with MDA-MB-231 cells was run by flow cytometry after treated with $0,0.1,1$ and $10 \mu \mathrm{mol} / \mathrm{L}$ ZINC20032678 for $48 \mathrm{~h}$. From the results we could see that the percentage of early apoptotic cells was $11.32 \%, 17.12 \%$ and $36.53 \%$ after treatment of $0.1,1$ and $10 \mu \mathrm{mol} / \mathrm{L}$ ZINC20032678, respectively; while the blank control did not induce obvious cell apoptosis (Figure 6). Therefore, the compound ZINC 20032678 could induce breast cancer cell apoptosis.
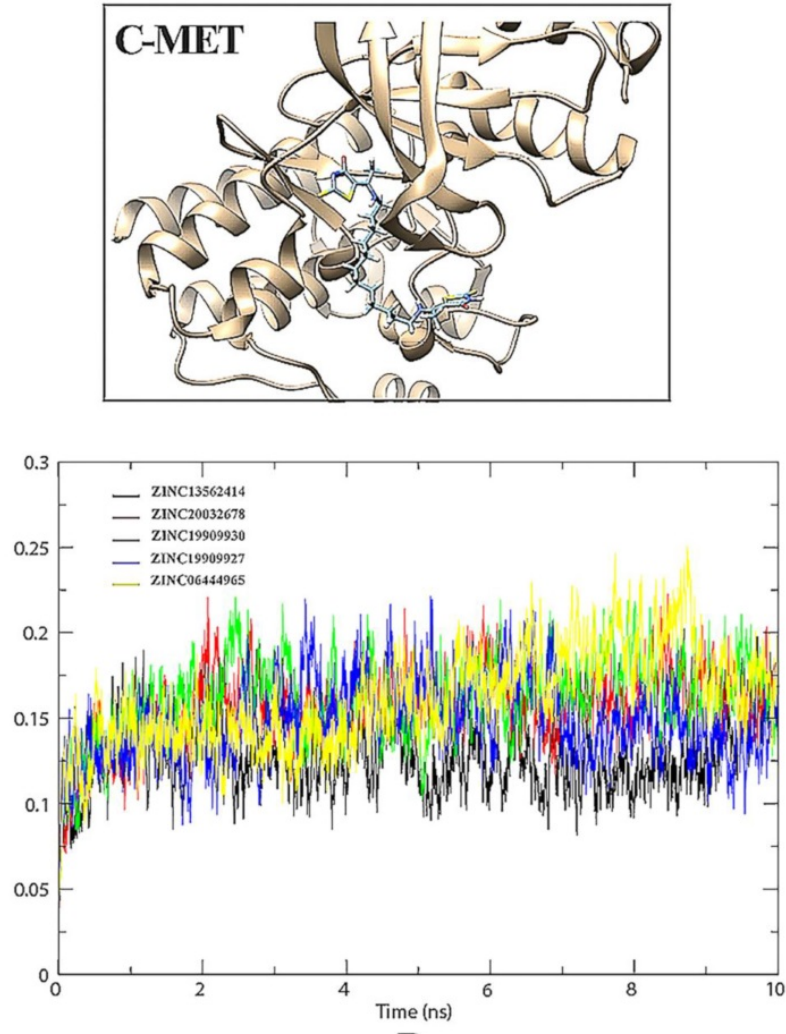

B

Figure 4. (A) Comparison of the binding site before and after docking of PARP-1 and its initial ligands. (B) Comparison of the binding site before and after docking of c-Met and its initial ligands. 
Table 2. Molecular docking results of c-Met/PARP-1 in complex with Specs products database

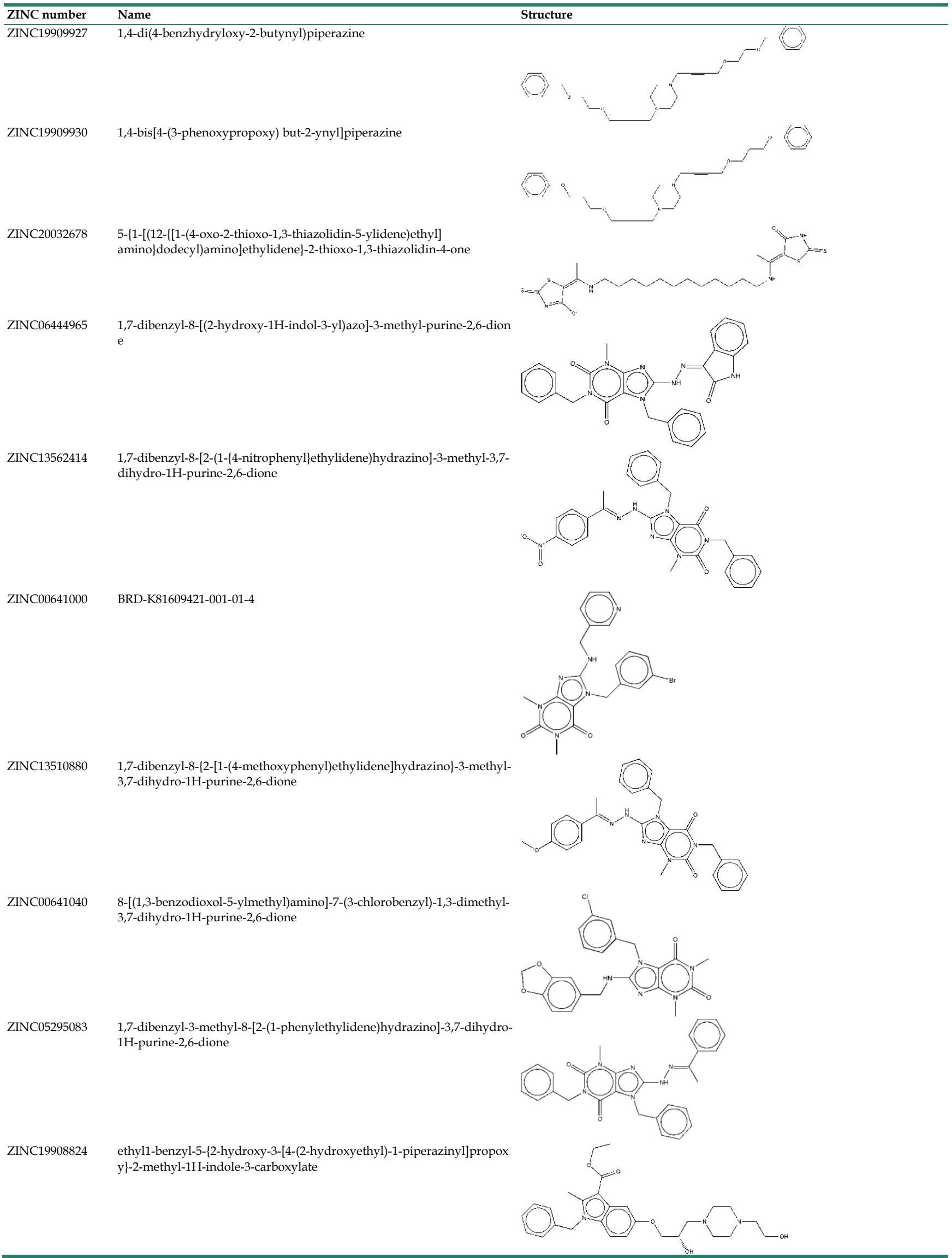


A

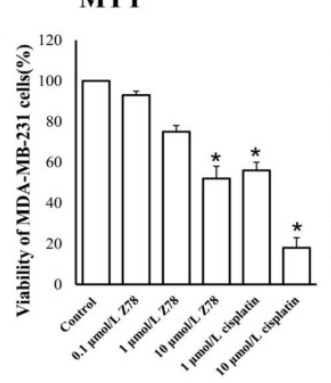

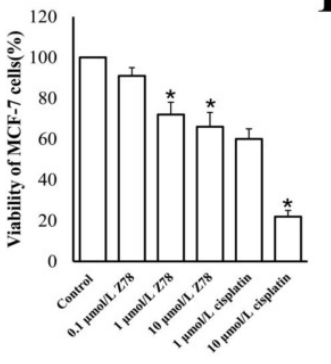

B

MCF-7 cells
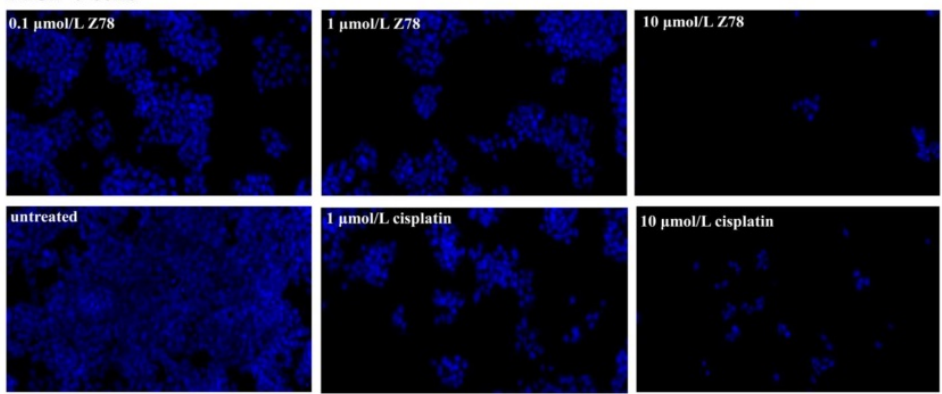

MDA-MB-231 cells
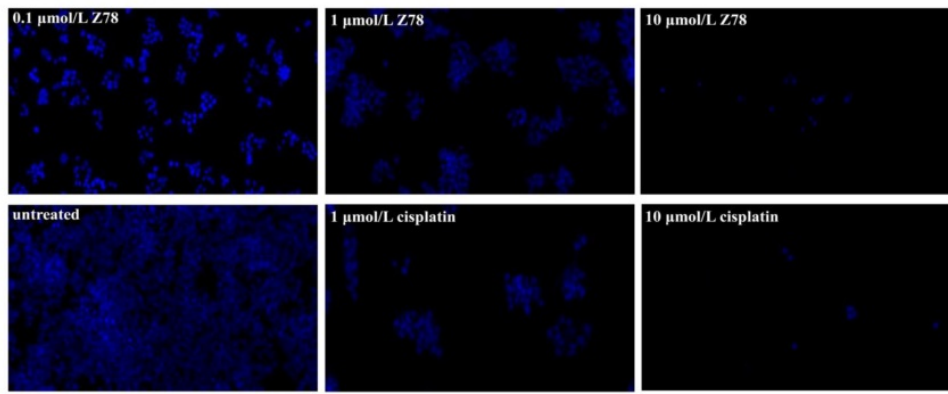

Figure 5. (A) The viability of breast cancer cells, MDA-MB-231 and MCF-7 cells, was detected by MTT assay. The cell growth was significantly inhibited by 10 umol/L ZINC20032678 at 48 h. (B) The Hoechst staining of MDA-MB-231 and MCF-7 cells treated with ZINC20032678 or cisplatin which induce cell death. Bar: X40. (C) The percentage of cell death according to Hoechst staining at 48 h. $* P<0.05$. Z78: ZINC20032678.

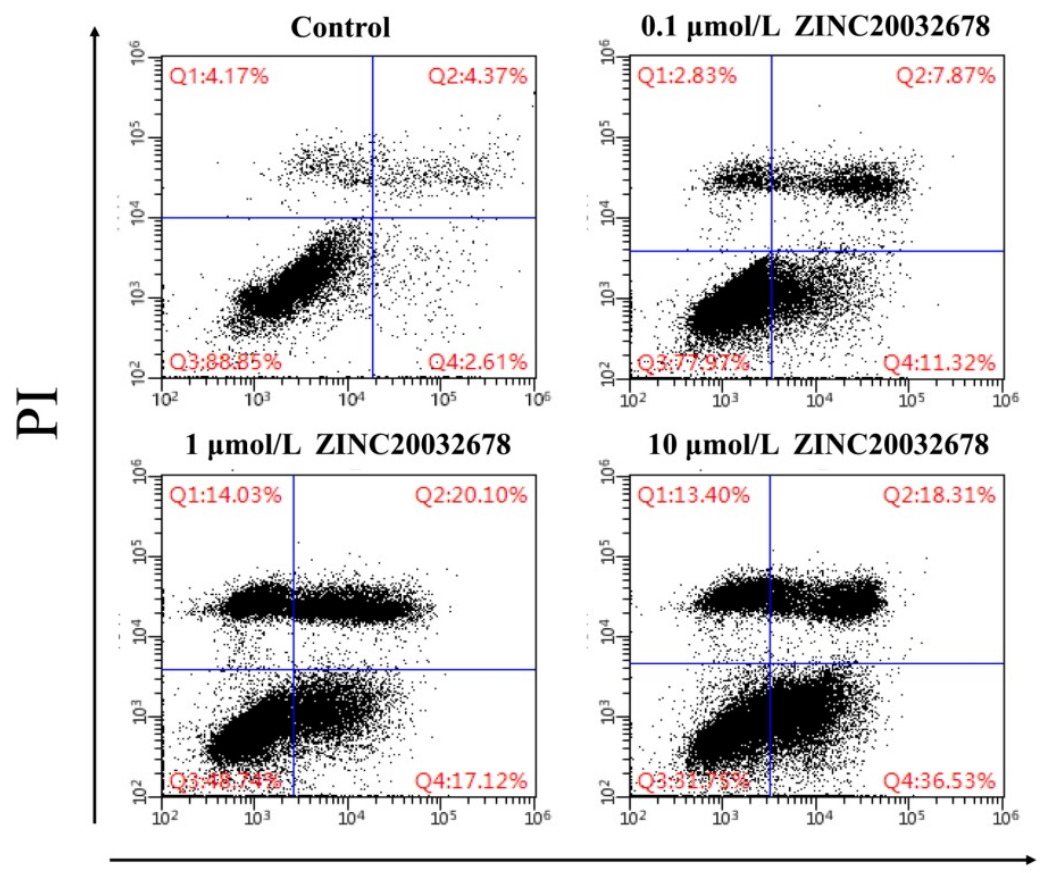

\section{Anneixin-V FITC-H}

Figure 6. Flow cytometry of Annexin V-FITC as a proxy for small molecular ZINC20032678 induced cellular apoptosis in MDA-MB-231 cells line for $48 \mathrm{~h}$. (A) There weren't obvious apoptosis in breast cancer cell lines in blank control group; (B) The percentage of early apoptosis cells was $11.43 \%$ in $0.1 \mu$ mol/L ZINC20032678 group; (C) The percentage of early apoptosis cells was $17.12 \%$ in $1 \mu \mathrm{mol} / \mathrm{L}$ ZINC20032678 group; (D) The percentage of early apoptosis cells was $36.53 \%$ in $10 \mu \mathrm{mol} / \mathrm{L}$ ZINC20032678 group.

\section{Inhibition of c-Met and PARP-1 kinases activities in vitro}

ZINC20032678 was identified to target both c-Met/PARP-1 proteins by series of in silico approaches. Therefore, the expression of phosphorylated c-Met and PARP-1 were detected by western blot, in which, the inhibitor of c-Met was crizotinib, and the inhibitor of PARP-1 was NMS-P118. In addition to this, there were two forms 
of PARP-1, full-length PARP-1 and cleaved PARP-1. The results showed that c-Met was significant inhibited by $10 \mu \mathrm{mol} / \mathrm{L}$ ZINC20032678 and its inhibitor; while ZINC20032678 and NMS-P118 disintegrated the full-length RARP-1 into cleaved PARP-1 (Figure 7A). Our results suggested that apoptosis could be induced in breast cancer cells through inhibition of c-Met/PARP-1 kinases activities.

After inhibition of c-Met/PARP-1 kinases activeties, the downstream apoptotic signaling pathways were then activated, the cleaved capase-3 was activated, and the expression of Bcl-2 was inhibited while the expression of Bax was up-regulated treated by ZINC20032678 or the corresponding inhibitors (Figure 7B). Therefore, ZINC20032678 could target both c-Met/PARP-1 sequentially activating the downstream apoptotic signaling pathway, eventually inducing breast cancer cell apoptosis.

\section{Discussion}

Of note, breast cancer is a heterogeneous disease which comprises a variety of pathologies and displays a range of histological characteristics and clinical outcomes. Systems biology approaches offer novel perspectives to reveal the molecular mechanisms of cancer, and thus shedding light on the identification of potential drug targets for future anti-cancer drug discovery [30,31]. Currently, the identification and validation of targeted therapy by using systems biology approaches are the most promising strategies in breast cancer research [32, 33]. Previous studies have indicated novel apoptotic kinase targets, namely AMPK and ZIPK, in cervical cancer by using systems biology methods. And, they screened and discovered a novel dual target activator targeting AMPK/ZIPK and inducing apoptosis in cervical cancer. Such small molecule named BL-AD008 is considered as a promising candidate agent in cervical cancer drug development [34]. In this study, multiple approaches including microarray analysis, biological characteristics were utilized to screen the hub proteins, and c-Met/PARP-1 were considered as the most effective drug targets in the context of breast cancer.

c-Met is an oncogene which encodes a membrane-bound tyrosine kinase implicated in the formation and/or progression of various types of tumors. It is reported that overexpression of c-Met has been discovered in a number of studies and with poor diagnosis in breast cancer [35]. Thus, inhibition of c-Met has become an emerging therapeutic strategy for breast cancer. Bulks of studies have highlighted the effects of c-Met deregulation on carcinogenesis and development of aggressive phenotypes in breast cancer [36]. Moreover, previous studies also indicated that combined therapy targeting both c-Met and EGFR may be beneficial for the treatment of breast cancer patients. Moreover, another study suggested c-Met overexpression was detected in $20-30 \%$ of breast cancer patients, which might be associated with a worse prognosis, suggesting that inhibition of c-Met might be an attractive oncology therapeutic approach [37]. Hitherto, a variety of small molecule kinase inhibitors targeting c-Met have been evaluated in different stages of clinical trials [38]. For example, Foretinib, a multi-target kinases inhibitor against both c-Met and VEGFR2, is currently under phase II trials [39]. Cabozantinib, an orally bioavailable tyrosine kinase inhibitor which targeting a number of tyrosine kinases, including RET, KIT and AXL, c-Met, etc., has been approved for the treatment of patients with progressive, metastatic medullary thyroid carcinoma $[40,41]$. However, novel inhibitors are still urgently waited for anti c-Met therapy in context of breast cancer.

PARPs are a family of nuclear protein enzymes which involved in a series of cellular responses, including DNA response, gene transcription, cell proliferation and apoptosis [42]. There are totally 18 family members in PARP family, and PARP-1 being the first to be characterized and most widely investigated. PARP-1 plays an important role in numerous cellular functions, such as transcriptional regulation and DNA damage repair. It is reported that PARP-1 is considered as a guardian angel against breast cancer [43]. Numerous clinical studies have
A

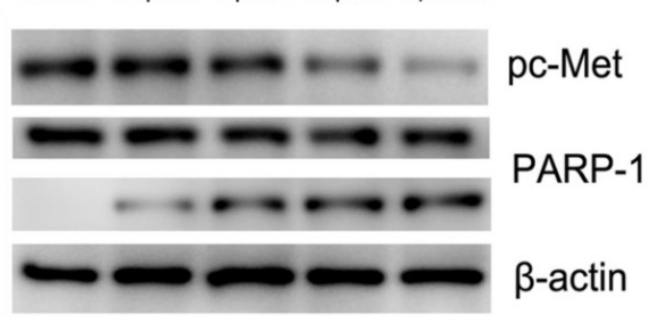

B Control $0.1 \mu \mathrm{MZ} \quad 1 \mu \mathrm{MZ} \quad 10 \mu \mathrm{M} \mathrm{Z} \quad 1 \mu \mathrm{M} \mathrm{INH}$

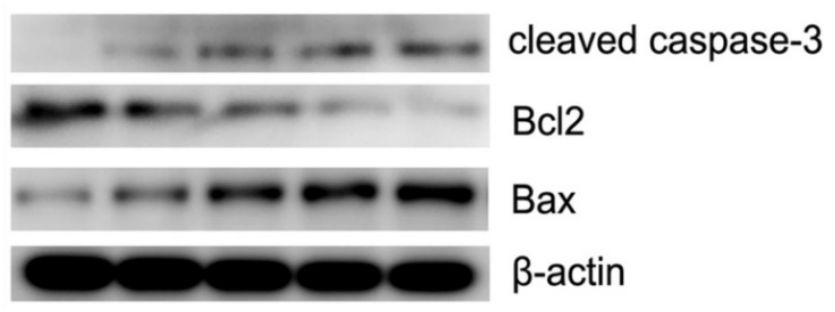

Figure 7. (A) The expression of phosphorylated c-Met, full-length and cleaved PARP-1 secreted by MDA-MB-231 cells at $72 \mathrm{~h}$ with western blot. (B) The expression of relative proteins of c-Met/PARP-1 downstream apoptotic signaling pathway, cleaved caspase-3, Bcl2 and Bax were detected by western blot. 
indicated that inhibiting PARP-1 made great effort towards personalizing treatment in breast cancer. To the best of our knowledge, there are more than 10 PARP inhibitors exerting promising treatment effect in clinical trials [44]. Interestingly, previous studies have also indicated that c-Met associated with and phosphorylated PARP-1 at Tyr907, and targeting both $t$ c-Met and PARP-1 could synergize to suppress the growth of breast cancer cells both in vitro and in vivo. Such study indicated that overexpression of c-Met may benefit from this combination therapy regardless of the cancer type [45].

In the current study, based on systems biology approaches and numerous literature researches, we manually selected c-Met and PARP-1 as the key targets in breast cancer. Subsequently, to identify inhibitors which could both targeting c-Met and PARP-1, molecular docking-based virtual screening was applied to filter 32791 candidate compounds, and MD was further calculated. Three small molecules, namely ZINC13562414, ZINC20032678, and ZINC 19909930 were recognized. We synthesized these molecules and performed in vitro study to confirm that ZINC20032678 was the most effective small molecule which could induce two breast cancer cell lines apoptosis through inhibition of c-Met/PARP-1 kinases activities. In a word, our discovered small molecule ZINC20032678 could both target the active sites of the c-Met/PARP-1 and thus inhibiting phosphorylation and recruitment of signaling effectors, the cleaved capase- 3 was activated; the expression of Bcl-2 was further inhibited while the expression of Bax was up-regulated, and eventually leading to breast cancer cell apoptosis. Therefore, ZINC20032678 could be considered as a promising and effective c-Met/PARP-1 inhibitor in future breast cancer drug discovery.

\section{Conclusions}

In this study, a series of bioinformatics approaches were performed to identify the hub proteins, and then c-Met and PARP-1 were identified as the vital targets in breast cancer. Subsequently, in vitro study was carried out to confirm that the small molecule named ZINC20032678 which could both targeting c-Met/PARP-1 and induced breast cancer cell apoptosis. Our discovered targets and agents would provide new clues for the applications and modifications of future cancer drug discovery from bench to clinic.

\section{Acknowledgments}

We thank Prof. Ling Li (Xihua University) for technological assistance. This work was supported in part by the National Natural Science Foundation of
China (81803561), the grants Scientific and Technological Funds for Young Scientists of Sichuan (2017JQ0060), the open fund project of Key Laboratory of Medicinal and Edible Plants Resources Development of Sichuan Education Department (10Y201804), the Chun hui Project of Ministry of Education (Z2015119), Department of Science and Technology of Sichuan (2018JY0348), Innovative Research Team in University of Sichuan (16TD0015), the International Cooperation grant from the Science and Technology Department of Sichuan (2019YFH 0054), the Scientific and Technological Funds of Sichuan (2018jy0222), and the scientific funds of health commission of Sichuan Province (19PJ001).

\section{Author Contributions}

Zheng Shi and Yong Tang conceived and designed the experiments; Lijia Cheng, Jie Chen, Hang Xiong, Xueling Yan, Guangya Xu, Tian Yu and Hui Zhou performed the experiments; Gang He and Hongbo Dong analyzed the data; Zheng Shi and Tian Yu wrote the paper.

\section{Competing Interests}

The authors have declared that no competing interest exists.

\section{References}

1. Siegel RL, Miller KD, Jemal A. Cancer Statistics, 2019. Ca A Cancer Journal for Clinicians. 2019,69(1):7-34.

2. Liu $Q$, Jin $C$, Wang $Y$, et al. Aptamer-conjugated nanomaterials for specific cancer cell recognition and targeted cancer therapy. NPG Asia Mater. 2014,6(4):e95.

3. Tang Y, Wang Y, Kiani MF, et al. Classification, treatment strategy, and associated drug resistance in breast cancer. Clin Breast Cancer. 2016,16(5):335-343.

4. Gaule PB, Crown J, O'Donovan N, et al. CMET in triple-negative breast cancer: Is it a therapeutic target for this subset of breast cancer patients?. Expert Opin Ther Tar. 2014,18(9):999-1009.

5. Schneider HC, Klabunde TJB. Understanding drugs and diseases by systems biology?. Bioorg Med Chem Lett. 2013,23(5):1168-1176.

6. Huang DW, Sherman BT, Lempicki RA. Bioinformatics enrichment tools: Paths toward the comprehensive functional analysis of large gene lists. Nucleic Acids Res. 2009;37(1):1-13.

7. Hiromi S, Seiichi I, Kyoko T, et al. New approaches to mechanism analysis for drug discovery using DNA microarray data combined with KeyMolnet. Current Drug Discovery Technologies. 2005,2(2):89-98.

8. Sherman BT, Lempicki RA. Systematic and integrative analysis of large gene lists using DAVID bioinformatics resources. NAT Protoc. 2009,4(1):44-57.

9. Szklarczyk D, Morris JH, Cook H, et al. The STRING database in 2017: Quality-controlled protein - protein association networks, made broadly accessible. Nucleic Acids Res. 2017,45(D1):D362-D368.

10. Gisterek I, Lata E, Halon A, et al. Prognostic role of c-met expression in breast cancer patients. Rep Pract Oncol Radiother. 2011,16(5):173-177.

11. Shi $\mathrm{Z}$, Sun $\mathrm{R}, \mathrm{Yu} \mathrm{T}$, et al. Identification of novel pathways in plant Lectin-Induced cancer cell apoptosis. INT J MOL SCI. 2016,17(2):228-228.

12. Du W, Elemento O. Cancer systems biology: Embracing complexity to develop better anticancer therapeutic strategies. Oncogene. 2015,34(25):3215-3225.

13. Berman HM, Westbrook J, Feng Z, et al. The protein data bank. Genetica. 2000,106(1-2):149-158.

14. Irwin JJ, Shoichet BK. ZINC--a free database of commercially available compounds for virtual screening. Journal of Chemical Information \& Modeling. 2005; 45(1):177-182.

15. Pettersen EF, Goddard TD, Huang CC, et al. UCSF Chimera--a visualization system for exploratory research and analysis. J Comput Chem. 2004,25(13):1605-1612.

16. Lang PT, Brozell SR, Mukherjee S, et al. DOCK 6: Combining techniques to model RNA-small molecule complexes. Rna-a Publication of the Rna Society. 2009,15(6):1219-1230 
17. Wang ZJ, Wan ZN, Chen XD, et al. In silico identification of novel kinase inhibitors by targeting B-Raf(v660e) from natural products database. J Mol Model. 2015,21(4): 102-102.

18. Shi Z, An N, Zhao S, et al. In silico analysis of molecular mechanisms of legume lectin-induced apoptosis in cancer cells. Cell Proliferat. 2013,46(1):86-96.

19. Graves AP, Shivakumar DM, Boyce SE, et al. Rescoring docking hit lists for model cavity sites: Predictions and experimental testing. J Mol Biol. 2008,377(3):914-934.

20. Fan J, Upadhye S, Worster A. Understanding receiver operating characteristic (ROC) curves. Cjem. 2006,8(1):19-20.

21. Sun $\mathrm{R}, \mathrm{Li} \mathrm{X}, \mathrm{Li} \mathrm{Y}$, et al. Screening of novel inhibitors targeting lactate dehydrogenase a via four molecular docking strategies and dynamics simulations. J Mol Model. 2015,21(5):133.

22. Pronk S, Páll S, Schulz R,et al.GROMACS 4.5: A high-throughput and highly parallel open source molecular simulation toolkit. Bioinformatics. 2013,29(7):845-854.

23. Hornak V, Abel R, Okur A, et al. Comparison of multiple Amber force fields and development of improved protein backbone parameters. Proteins-structure Function \& Bioinformatics. 2010,65(3):712-725.

24. Jakalian A, Jack DB, Bayly CI. Fast, efficient generation of high-quality atomic charges. AM1-BCC model: II. Parameterization and validation. J Comput Chem. 2002,23(16):1623-1641.

25. Wang J, Wang W, Kollman PA, et al. Automatic atom type and bond type perception in molecular mechanical calculations. J Mol Graph Model. 2007; 25(2):247-260.

26. Wang J, Wolf RM, Caldwell JW, et al. Development and testing of a general amber force field. J Comput Chem. 2004,25(9):1157-1174.

27. Schoenberg R, Arminger G. LINCS: Linear convariance structure analysis. Multivar Behav Res. 1988,23(2):271-273.

28. Kollman PA, Massova I, Reyes C, et al. Calculating structures and free energies of complex molecules: Combining molecular mechanics and continuum models. Cheminform. 2010,32(10):889-897.

29. Harvey MJ, De FG. An implementation of the smooth particle mesh ewald method on GPU hardware. Journal Of Chemical Theory \& Computation. 2009,5(9):2371-2377.

30. Hoyen CM, Jones JL, Kermorgant S. The clinical and functional significance of c-Met in breast cancer: A review. Breast Cancer Res. 2015,17(1):1-11.

31. Shi Z, Chen J, Guo X, et al. In silico identification of potent small molecule inhibitors targeting epidermal growth factor receptor 1. Journal Of Cancer Research \& Therapeutics. 2018,14(1):18-18.

32. Radvanyi L. Targeting the cancer mutanome of breast cancer. Nat Med. 2018,24(6):703-704.

33. Costa RLB, Han HS, Gradishar WJ. Targeting the PI3K/AKT/mTOR pathway in triple-negative breast cancer: A review. Breast Cancer Research \& Treatment. 2018,169(3):347-406.

34. Fu L, Zhang S, Zhang L, et al. Systems biology network-based discovery of a small molecule activator BL-AD008 targeting AMPK/ZIPK and inducing apoptosis in cervical cancer. Oncotarget. 2015,6(10):8071-8088.

35. Kim YJ, Choi JS, Seo J, et al. MET is a potential target for use in combination therapy with EGFR inhibition in triple-negative/basal-like breast cancer. INT J Cancer. 2014,134(10):2424-2436.

36. Erlmeier F, Ivanyi P, Hartmann A, et al. C-Met in chromophobe renal cell carcinoma. Med Oncol. 2017,34(2):15-15.

37. Stanley A, Ashrafi GH, Seddon AM, et al. Synergistic effects of various Her inhibitors in combination with IGF-1R, C-MET and Src targeting agents in breast cancer cell lines. Sci Rep-Uk. 2017,7(1):3964-3964.

38. Yi YW, You K, Bae EJ, et al. Dual inhibition of EGFR and MET induces synthetic lethality in triple-negative breast cancer cells through downregulation of ribosomal protein S6. Int J Oncol. 2015,47(1):122-132.

39. Logan TF. Foretinib (XL880): C-MET inhibitor with activity in papillary renal cell cancer. Curr Oncol Rep. 2013,15(2):83-90.

40. Sun Y, Sun L, An Y, et al. Cabozantinib, a novel c-Met inhibitor, inhibits colorectal cancer development in a xenograft model. Medical Science Monitor International Medical Journal Of Experimental \& Clinical Research. 2015,21:2316-2321.

41. Shi Z, Yu T, Sun R, et al. Discovery of novel human epidermal growth factor receptor-2 inhibitors by structure-based virtual screening. Pharmacogn MAG. 2016,12(46):139-144.

42. Weaver AN, Yang E. Beyond DNA repair: Additional functions of PARP-1 in cancer. Front Oncol. 2013,3:290-290.

43. Sánchez-Muñoz A, Pérez-Ruiz E, Jiménez B, et al. Targeted therapy of metastatic breast cancer. Clin Transl Oncol. 2009,11(10):643-650.

44. Yi D, Yamaguchi H, Wei Y, et al. Blocking c-Met-mediated PARP1 phosphorylation enhances anti-tumor effects of PARP inhibitors. Nat Med. 2016,22(2):194-201.

45. Sheeba I, Alan A, Andrew T. Therapeutic potential of PARP inhibitors for metastatic breast cancer. Expert Rev Anticancer Ther. 2011,11(8):1243-1251. 